\title{
TABLE OF CONTENTS
}

Table of Contents..............................................................................

Acknowledgments ......................................................................... vii







Chapter One. Hick's Use of Irenaeus.................................................23





“Augustinian” Theodicy ...........................................................



Hick's Irenaeus …………………………………………….........

Hick's "Irenaean" Theodicy ........................................................... 40

Creation and Progression............................................................ $4 \mathrm{I}$







Chapter Two. Background and Sources for Irenaeus on the

Problem of Evil ......................................................................... 49

Introduction.......................................................................... 49

The Problem of Evil in Pagan Thought ....................................50

The Problem of Evil in Early Christian Thought.......................54







Chapter Three. Irenaeus and Theodicy............................................. 79










Diversity within Unity .............................................................. 90

Non-Speculative beyond Scripture............................................... 98

The Thought of Irenaeus's Opponents on the Problem of









Other Opponents ............................................................................ 109

Conclusion .................................................................................... 109

Irenaeus on the Problem of Evil................................................. I09

Irenaeus on Creation ......................................................................II

Irenaeus on Humanity's Progression in the One Plan or Economy of God ....................................................................14

Irenaeus on The Origin of Evil ...................................................116

Irenaeus on Human Transgression ...............................................19

Irenaeus on Death ..........................................................................121

Irenaeus's Free-Will Defense.........................................................123



Chapter Four. The Divergence of Hick's Interpretation ................. I27



Three Key Differences in Hick's Reading ............................... I2 28

Creation as Including Divinely Created Evil............................... I28

An Excusable Disobedience, Not a Devastating Tragedy .............132

Two Stages with One Method of Humanity's

Progression ........................................................................135

Hick's False Dichotomy ......................................................... 139

Conclusion ......................................................................... 145

Chapter Five. Conclusion................................................................ 147







Articles and Essays ………………….................................. 172

Unpublished Materials ............................................................ I9I

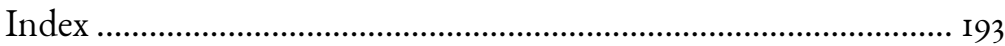

